\title{
Association between $I L-27$ Gene Polymorphisms and Cancer Susceptibility in Asian Population: A Meta-Analysis
}

\author{
Abdolkarim Moazeni-Roodi ${ }^{1,2}$, Mohammad Hashemi ${ }^{3,4 *}$, Saeid Ghavami ${ }^{2,5,6 *}$
}

\begin{abstract}
Background: Interleukin 27 (IL-27) has potent antitumor activity. Several epidemiological studies have designated that genetic variants of the IL-27 gene may contribute to various cancer susceptibility, but the data were inconclusive. Objective: The current meta-analysis aimed to address the association between $I L-27$ rs 153109 , rs 17855750, and rs 181206 polymorphisms and the risk of cancer. Data Sources: Our team has selected eligible studies up to May 1, 2020, from several electronic databases, including Web of Science, PubMed, Scopus, and Google Scholar databases. Results: Our meta-analysis revealed that the carriers $r s 153109 A>G$ polymorphism in the $I L-27$ gene have higher risks of diseases in the heterozygous $(\mathrm{OR}=1.26,95 \% \mathrm{CI}=1.06-1.49, \mathrm{P}=0.007, \mathrm{AG}$ vs $\mathrm{AA})$, homozygous $(\mathrm{OR}=1.18$, $95 \% \mathrm{CI}=1.01-1.37, \mathrm{p}=0.33, \mathrm{GG}$ vs AA $)$, dominant $(\mathrm{OR}=1.25,95 \% \mathrm{CI}=1.07-1.47, \mathrm{P}=0.006, \mathrm{AG}+\mathrm{GG}$ vs AA $)$, and allele $(\mathrm{OR}=1.15,95 \% \mathrm{CI}=1.04-1.27, \mathrm{P}=0.008, \mathrm{G}$ vs $\mathrm{A})$ genetic models. Stratified analysis by cancer type indicated that this variant was significantly associated with gastrointestinal cancer, colorectal cancer and breast cancer. The findings did not support an association between $r s 17855750 T>G, r s 181206 T>C$ polymorphisms of $I L-27$ and cancer risk. Conclusion: the current study findings suggest that IL-27 rs153109 polymorphism significantly increased the risk of cancer susceptibility. Well-designed replication in a larger independent genetic association study with larger sample sizes in diverse ethnicities is required to verify the findings.
\end{abstract}

Keywords: $I L-27$ - polymorphism- cancer- meta-analysis

Asian Pac J Cancer Prev, 21 (9), 2507-2515

\section{Introduction}

Cancer, a major public health concern, remains a leading cause of morbidity and mortality worldwide (Siegel et al., 2015). While the etiologies of cancer is complicated and not fully understood, growing evidences indicating that a complex interaction between genetic and environmental factors involved in cancer development (Lichtenstein et al., 2000).

Interleukin-27 (IL-27), belonging to the IL-12 family, is a heterodimeric cytokine comprising of two subunits, $1 \mathrm{~L}-27 \mathrm{p} 28$ and the Epstein-Barr virus-induced gene 3 protein (EBI3), and is generally secreted by activated antigen-presenting cells (Devergne et al., 1996; Liu et al., 2008). The human $\mathrm{Il}-27$ gene (IL-27P28) is located on chromosome 16 (16p11) (Pflanz et al., 2002). It is well-known that IL-27 possesses antitumor activities against a variety of tumor types (Nagai et al., 2010; Di
Carlo et al., 2014; Yoshida and Hunter, 2015; Yoshimoto et al., 2015). IL-27 is a polymorphic gene and several studies examined the association between $I L-27$ gene polymorphisms and risk of various cancers including, non-small-cell lung cancer (NSCLC) (Ge and Xiao, 2016), acute lymphoblastic leukemia (ALL) (Ghavami et al., 2018), nasopharyngeal carcinoma (NPC) (Wei et al., 2009; Pan et al., 2012), colorectal cancer (CRC) (Guo et al., 2012; Huang et al., 2012; Lyu et al., 2015), prostate cancer (PCa) (Munretnam et al., 2014), papillary thyroid carcinoma (PTC) (Zhang et al., 2015; Nie et al., 2017), hepatocellular carcinoma (HCC) (Peng et al., 2013), renal cell carcinoma (RCC) (Pu et al., 2015), osteosarcoma (Tang et al., 2014), esophageal cancer (Tao et al., 2012), cervical cancer (Wang et al., 2016), endometrial cancer (Yu et al., 2016), ovarian cancer (Zhang et al., 2014b), breast cancer (Zhang et al., 2014a), glioma (Zhao et al., 2009), and bladder cancer (Zhou et al., 2015). However, 
the findings of these studies have been controversial. So we conducted the present meta-analysis of eligible published studies to further assess the association between the $I L-27$ polymorphisms and cancer risk.

\section{Materials and Methods}

\section{Identification of Eligible Studies}

Two authors independently carried out a systematic literature search in PubMed, Web of Knowledge, and Scopus for all related reports using the key words " $I L$ 27 or IL27 or interleukin 27" and "polymorphism or SNP or variation" and "cancer or tumor or carcinoma or malignancy or neoplasm". The last search was updated on March 04, 2019.

\section{Inclusion and Exclusion Criteria}

Studies were implemented in the current meta-analysis if they met all of the criteria: (1) Assessment of the relationship between IL-27 gene polymorphisms and cancer susceptibility; (2) Case-control studies; (3) Adequate data to estimate pooled ORs with a $95 \%$ CIs. The exclusion criteria were: (1) not a case-control study, reviews, case reports, meta-analysis, and comments; (2) duplicate publication; (3) studies with insufficient data.

\section{Data extraction}

The data extraction from the eligible studies was achieved independently by two researchers according to the inclusion and exclusion criteria mentioned above. In each study, the following items were collected from each study: first author's name, publication year, country, ethnicity, cancer type, source of controls, total number of cases and controls, genotype distributions of cases and controls, and Hardy-Weinberg equilibrium (HWE), respectively.

\section{Statistical analysis}

All statistical analyses were achieved using Stata, version 14.1 (Stata Corporation, College Station, TX, USA).

The HWE was evaluated for each study by the chi-square test in the control group. Pooled ORs and corresponding $95 \% \mathrm{CIs}$ were calculated to estimate the strength of association between IL-27 gene polymorphism and cancer risk. The significance of the pooled OR was determined by $\mathrm{Z}$ test, in which $\mathrm{p}$-value less than 0.05 was considered statistically significant.

The Q statistic test was used to check the heterogeneity among studies included in the meta-analysis. A $p>0.10$ indicated a lack of heterogeneity among studies, consequently the fixed effect model was used to calculate pooled OR. Otherwise, a random effects model was utilized.

Publication bias was evaluated by Begg's funnel plot qualitatively, and Begg's and Egger's tests quantitatively. $\mathrm{P}$-value less than 0.05 considered significant publication bias.

Sensitivity analysis was done by removing each study in turn to measure the results stability.

\section{Results}

\section{Study Characteristics}

In the current study, according to the inclusion and exclusion criteria, ultimately 20 case-control studies included in the meta-analysis. For $r s 153109,21$ studies containing 6,331 cases and 7,287 controls for were included in the quantitative analysis. Regarding rs 17855750 variant, 4,023 cases and 4,671 controls from 14 studies and for $r s 181206$ polymorphism, 2,078 cases and 2,242 controls from were 7 studies were included in the meta-analysis. The characteristics of the included studies are summarized in Table 1, Table 2 and Table 3.

Association between IL-27 polymorphisms and cancer risk The frequency distribution of genotype and allele of the $I L-27$ polymorphisms in cases and controls are indicated in Table 1, Table 2 and Table 3. Table 4 shows the main findings of our meta-analysis. Regarding $r s 153109 A>G$ variant, 21 independent studies were pooled and a random effect was applied due to the presence of significant heterogeneity. The finding revealed that $r$ s 153109 variant significantly increased the risk of cancer in heterozygous $(\mathrm{OR}=1.26,95 \% \mathrm{CI}=1.06-1.49, \mathrm{P}=0.007, \mathrm{AG}$ vs AA), homozygous $(\mathrm{OR}=1.18,95 \% \mathrm{CI}=1.01-1.37, \mathrm{p}=0.33, \mathrm{GG}$ vs $\mathrm{AA})$, dominant $(\mathrm{OR}=1.25,95 \% \mathrm{CI}=1.07-1.47, \mathrm{P}=0.006$, $\mathrm{AG}+\mathrm{GG}$ vs AA), and allele $(\mathrm{OR}=1.15,95 \% \mathrm{CI}=1.04-1.27$, $\mathrm{P}=0.008$, G vs A) genetic models (Figure 1 and Table 4).

Stratified analysis by cancer type (Table 5) revealed that rs 153109 significantly increased the risk of gastrointestinal (GI) cancer in homozygous $(\mathrm{OR}=1.35,95 \% \mathrm{CI}=1.11$ $1.65, \mathrm{p}=0.003)$, dominant $(\mathrm{OR}=1.28,95 \% \mathrm{CI}=1.02-1.59$, $\mathrm{p}=0.030)$ and allele $(\mathrm{OR}=1.18,95 \% \mathrm{CI}=1.07-1.30$, $\mathrm{p}=0.007)$ genetic models. Besides, the variant was significantly associated with colorectal cancer (CRC) and breast cancer susceptibility in all genetic model tested (Table 5).

The findings showed that $\mathrm{rs} 17855750 \mathrm{~T}>\mathrm{G}$, and rs $181206 \mathrm{~T}>\mathrm{C}$ variants were not associated with cancer risk (Table 4).

\section{Heterogeneity and publication bias}

Heterogeneity among studies involved in the meta-analysis is presented in Table 4. The findings indicated that heterogeneity exist among studies and random-effects was used to estimate the pooled OR and 95\% CI (Figure 2 and Table 4).

Begg's funnel plot, Begg's test, and Egger's test (Figure 3, and Table 4) indicated no evidence of significant publication bias.

\section{Sensitivity analysis}

After doing the sensitivity analyses, the pooled ORs showed no statistically significant changes in heterozygous, dominant, recessive, and allele representing that our findings are stable and reliable in overall analysis (Figure 4).

\section{Discussion}

Several studies examined the association between 


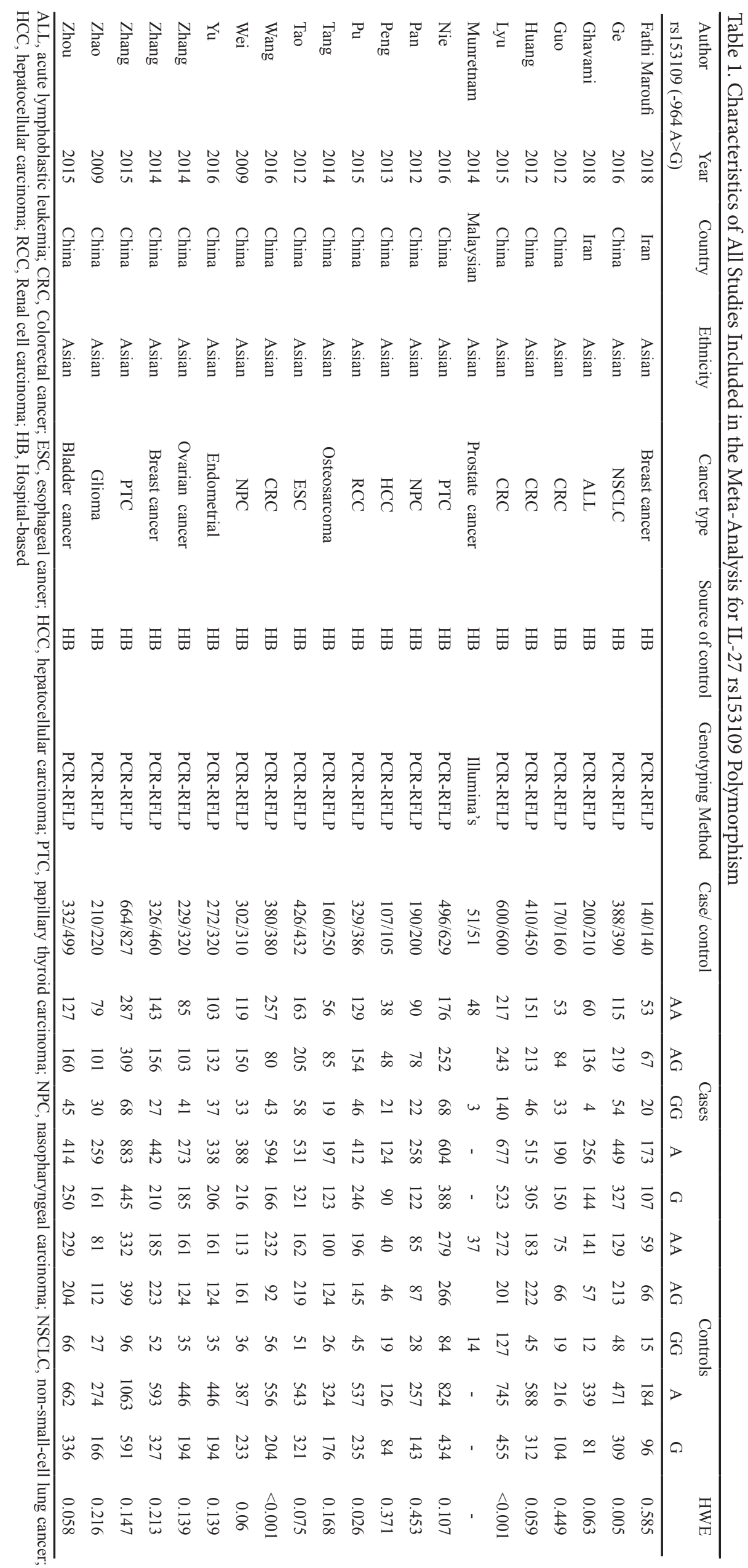




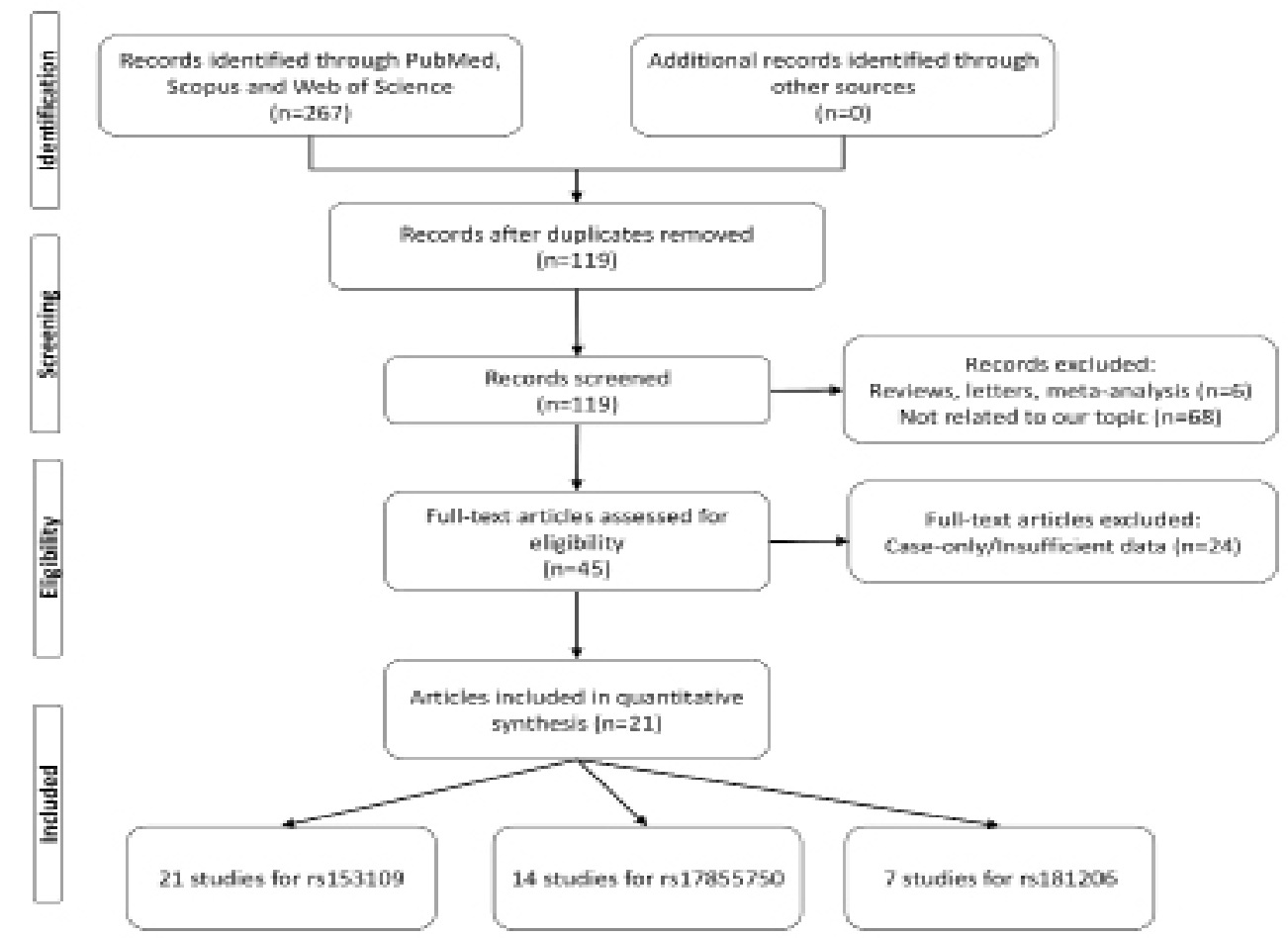

Figure 1. The Flow Diagram of Screening and Study Selection for Meta-Analysis

IL-27 polymorphisms and the risk of various cancer (Wei et al., 2009; Zhao et al., 2009; Guo et al., 2012; Huang et al., 2012; Pan et al., 2012; Tao et al., 2012; Peng et al., 2013; Munretnam et al., 2014; Tang et al., 2014; Zhang et al., 2014a; Zhang et al., 2014b; Lyu et al., 2015; Pu et al., 2015; Zhang et al., 2015; Zhou et al., 2015; Ge and Xiao, 2016; Wang et al., 2016; Yu et al., 2016; Nie et al., 2017; Ghavami et al., 2018). The findings were controversial, though it is difficult to clarify the inconsistent findings. In this study, we conducted a comprehensive meta-analysis of all eligible studies to derive a more precise estimation of the relationship between $I L-27$ polymorphism and cancer risk. After pooling all the available data, the finding suggested that $I L-27$ rs 153109 (-964 A > G) significantly increased the risk of overall cancer. Stratified analysis by cancer type designated that rs 153109 polymorphism

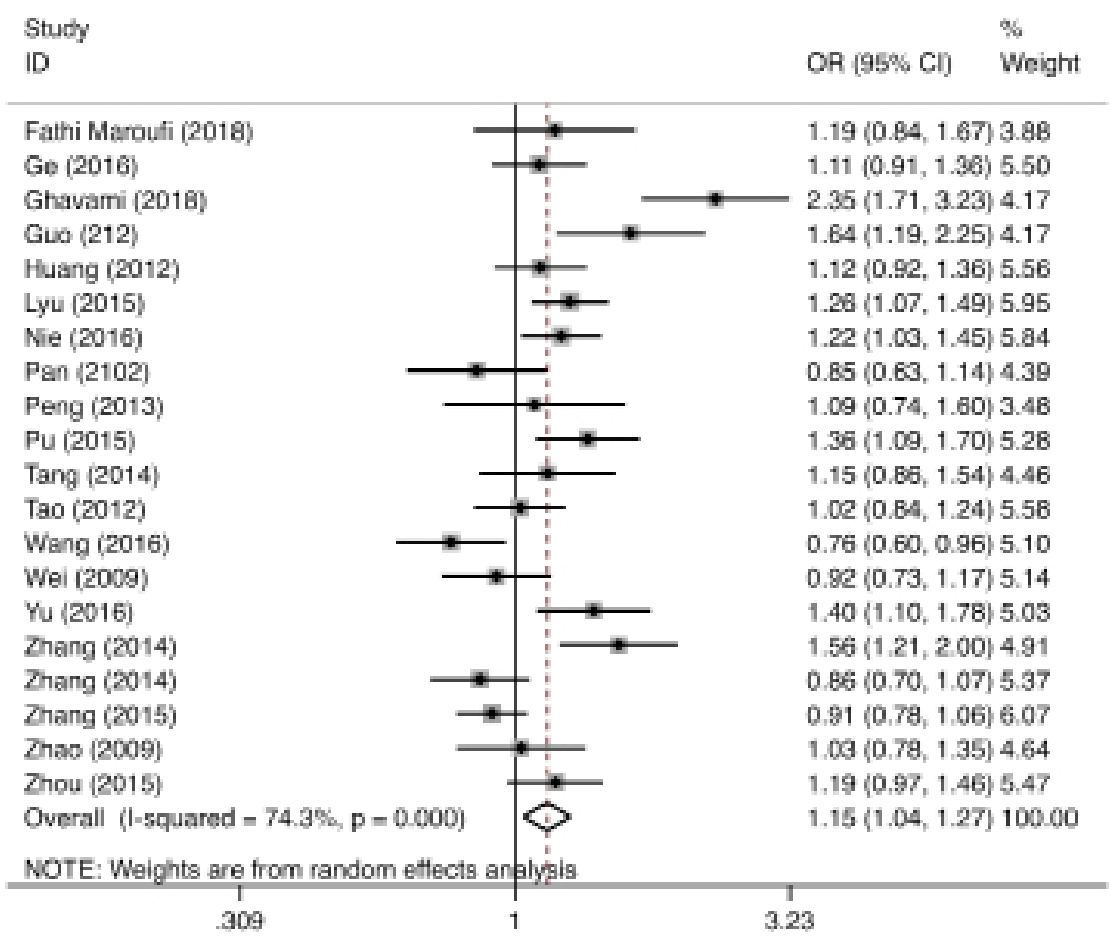

Figure 2. The Forest Plot for Association between rs153109 A $>$ G Polymorphism in the IL-27 and Cancer Susceptibility for $\mathrm{G}$ vs A. 


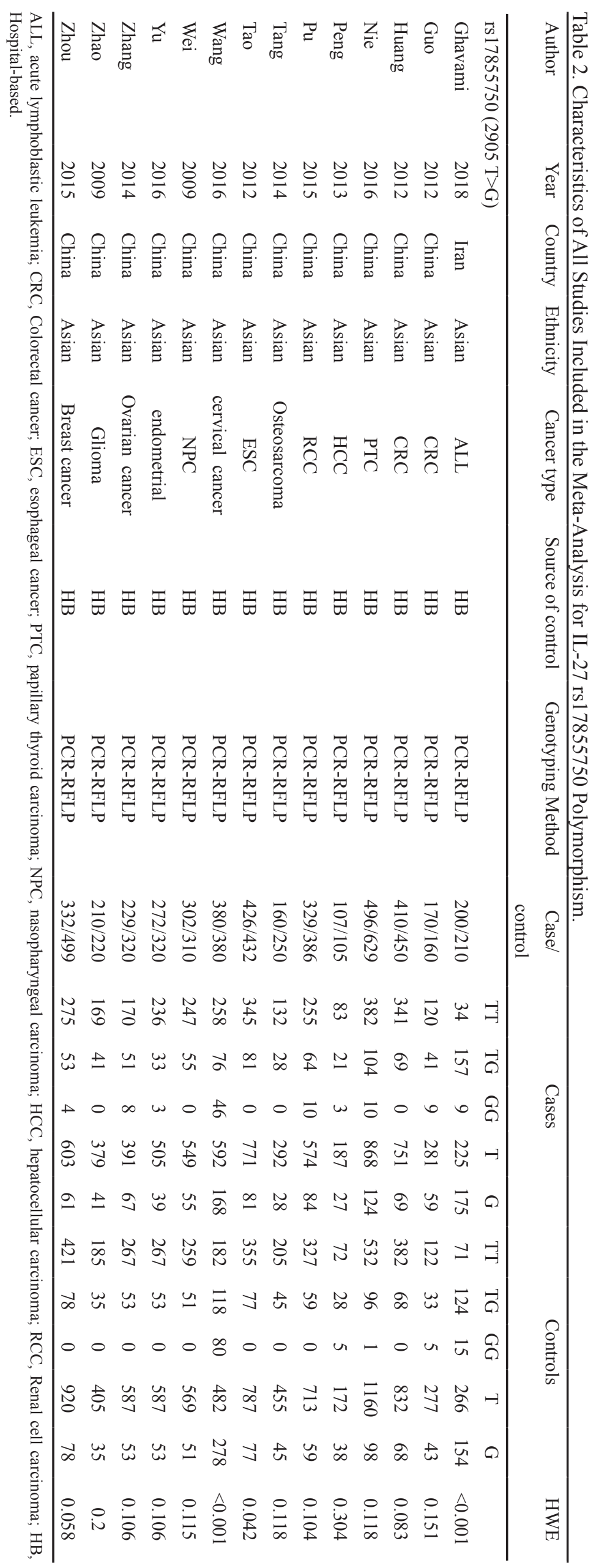




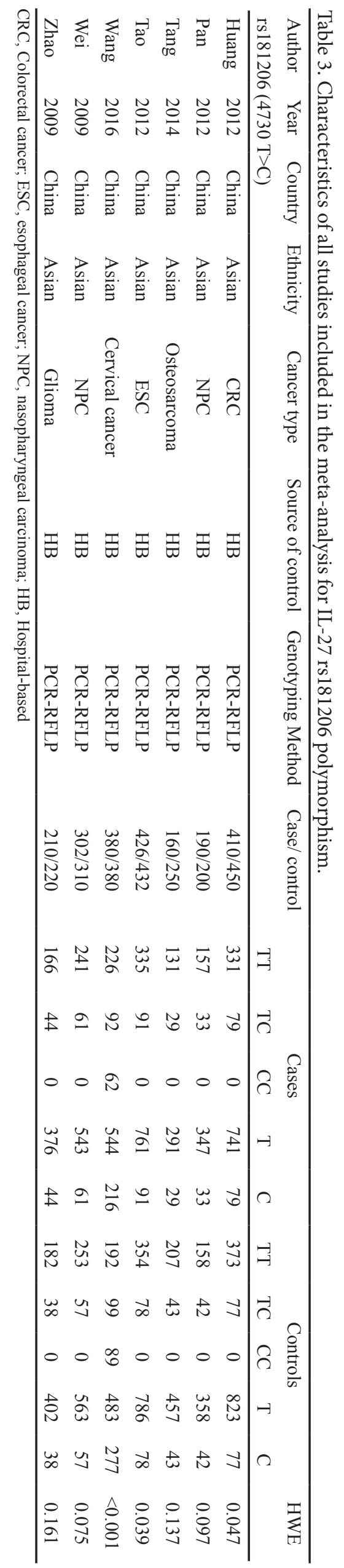

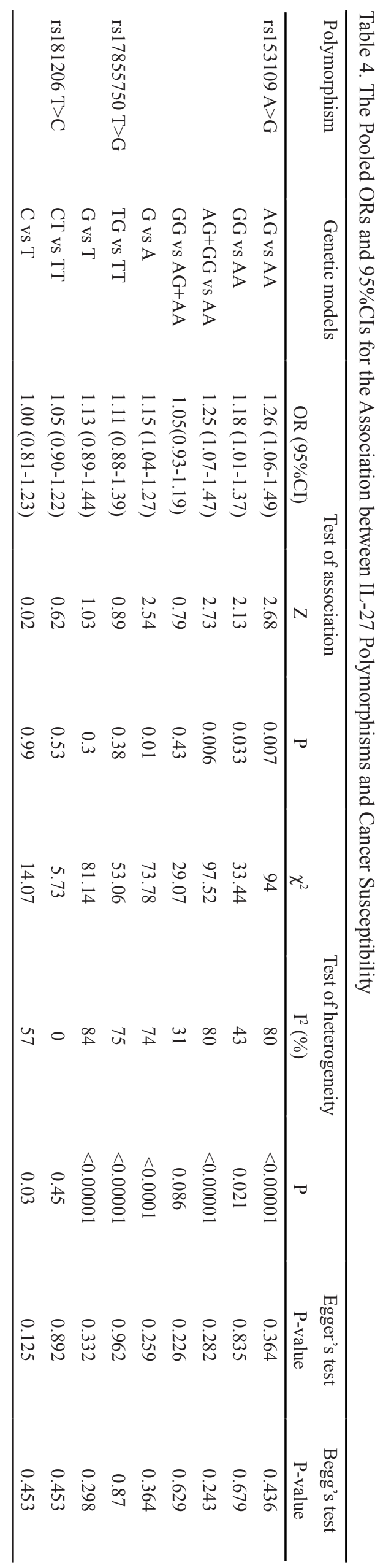




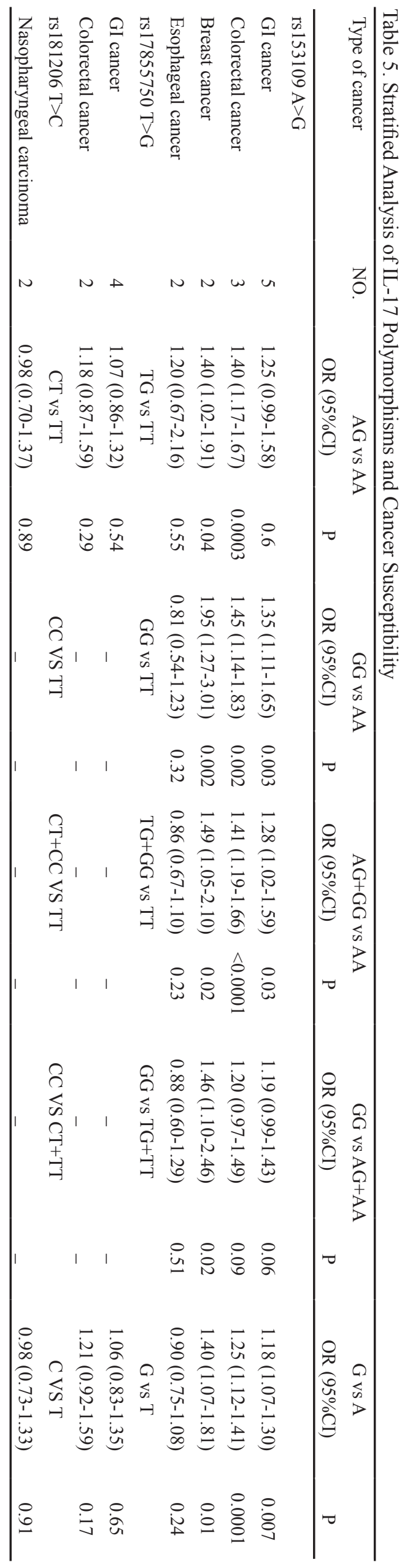

was positively associated with GI cancer, CRC and BC susceptibility. The findings did not support an association between $r s 17855750(2905 \mathrm{~T}>\mathrm{G})$ and $r s 181206$ (4730 $\mathrm{T}>\mathrm{C}$ ) polymorphisms and cancer susceptibility.

The molecular mechanisms by which variant increased the risk of cancer have not been clarified. It seems reasonable to speculate that rs 153109 polymorphism effects on $I L-27$ expression. There is increasing evidence that the expression level of $I L-27 p 28$ gene is decreased in various cancers including epithelial ovarian cancer (Zhang et al., 2014b), bladder cancer (Zhou et al., 2015), esophageal cancer (Tao et al., 2012), osteosarcoma (Tang et al., 2014), as well as papillary thyroid cancer (Zhang et al., 2015). It has been shown that $I L-27$ differentially regulates the expression of several microRNAs (miR) such as hsa-miR-7702, hsa-miR-7704, hsa-miR-7704 hsa-miR-6852, and hsa-miR-6852 (Swaminathan et al., 2013; Poudyal et al., 2018).

Cytokines, secreted by cells of innate and adaptive immune systems, are small proteins that play key roles in immune responses. $I L-27$ is produced early after activation by antigen-presenting cells, including monocyte-derived dendritic cells and lipopolysaccharidestimulated monocytes (Chiyo et al., 2004; Owaki et al., 2005). IL-27 mediates its biological functions via a heterodimeric receptor consisting of WSX-1 and glycoprotein 130 (gp130) (Pflanz et al., 2004). Binding of $I L-27$ to its receptor activates Janus kinase (JAK)signal transducer and activator of transcription (STAT) and mitogen-activated protein kinase (MAPK) signaling (Kastelein et al., 2007). IL-27 has potent antitumor activity (Hisada et al., 2004; Chiyo et al., 2005). It exerts antitumor activity by promoting the generation of myeloid progenitor cells that can differentiate into M1 macrophages (Chiba et al., 2018). In addition, $I L-27$ synergizes with $I L-12$ to potentiate IFN- $\gamma$ production by activated naive T-cell and natural killer-cell populations (Pflanz et al., 2002). Beside, $I L-27$ is a major stimulus of $I L-10$ production by $\mathrm{T}$ cell (Hunter and Kastelein, 2012; Liu et al., 2013).

Some limitations should be addressed in our metaanalysis. First, heterogeneity among studies was observed which may be result of difference of ethnicity, source of control, and cancer type. Second, this study focused on the impact of limited variants of $I L-27$ and cancer susceptibility. Gene-gene as well as gene-environment interactions could influence cancer risk. Third, all studies were from Asian populations; consequently, conclusions drawn may not apply to the all population. Finally, the sample sizes of the studies are relatively small particularly in subgroup analysis. So, the results should be interpreted with caution.

In conclusion, the findings of this meta-analysis provide evidence for an association between $I L-27$ rs 153109 polymorphism and cancer risk. Well-designed studies with larger sample sizes in various cancer and different ethnicities are still needed in the future.

\section{Acknowledgments}

The authors like to acknowledge the Zahedan University of Medical Sciences research office to provide Asian Pacific Journal of Cancer Prevention, Vol 212513 


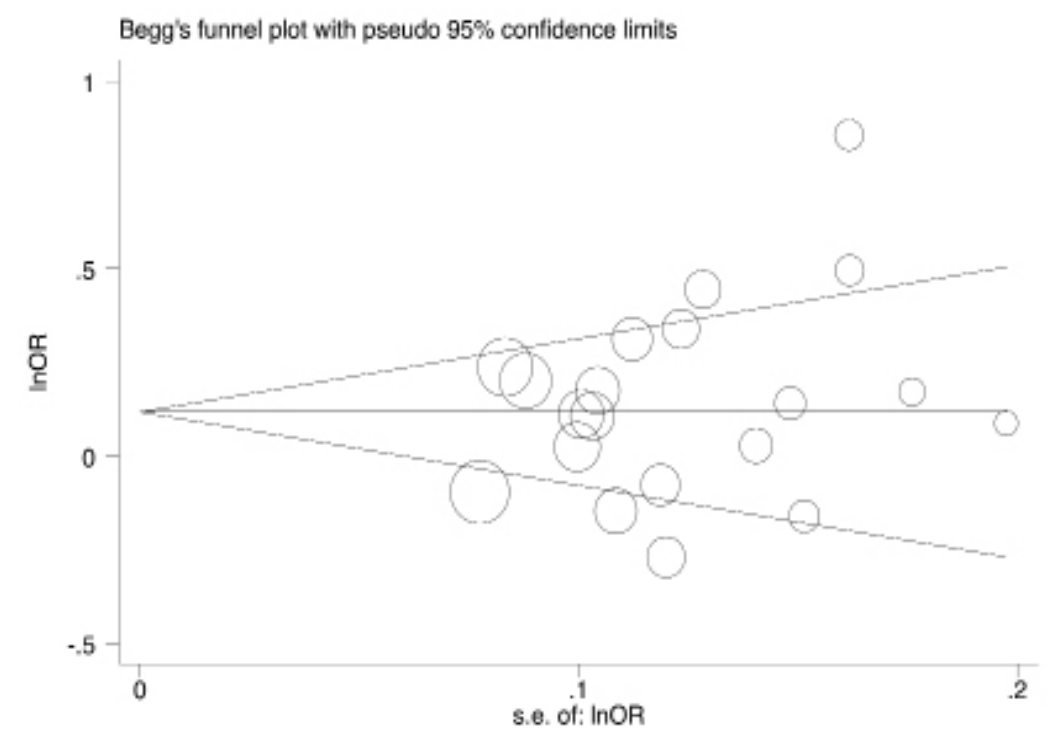

Figure 3. Begg's Funnel Plot for Publication Bias Test for IL-27 rs153109 A >G Polymorphism and Cancer Risk for G vs A.

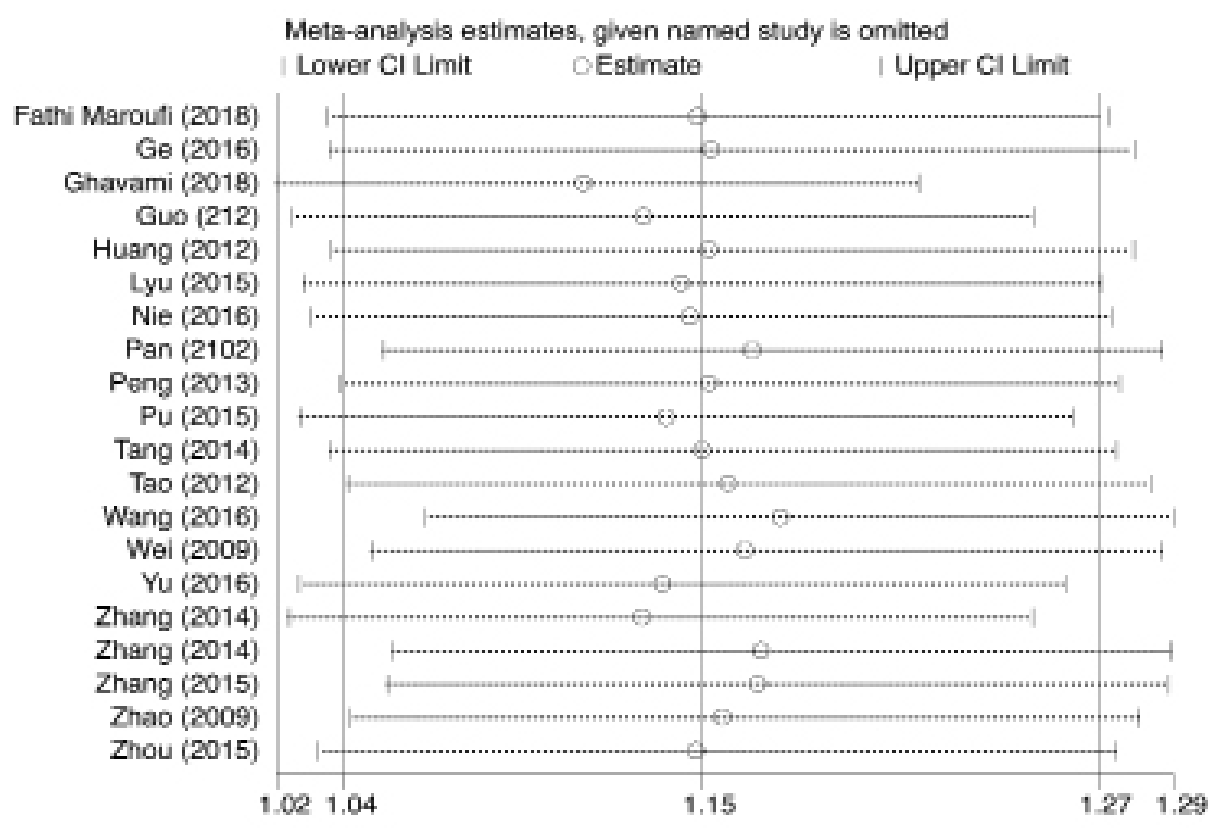

Figure 4. Sensitivity Analyses for Association between IL-27 rs153109 A>G Polymorphism and Cancer Risk for G vs A.

logistics for the literature search. The work was not supported by any operating grant and fund and was not a part of any graduate and under graduate thesis.

We would like to dedicate this article to Professor Mohamad Hashemi who passed away recently after the submission of this work. He was a pioneer in genetic studies.

\section{Acknowledgments}

We would like to dedicate this article to Professor Mohamad Hashemi who passed away recently after the submission of this work. He was a pioneer in genetic studies.

\section{Conflict of interest}

The authors declare that they have no conflict of interest.

\section{References}

Chiba Y, Mizoguchi I, Furusawa J, et al (2018). Interleukin-27 Exerts its antitumor effects by promoting differentiation of hematopoietic stem cells to M1 macrophages. Cancer Res, 78, 182-94.

Chiyo M, Shimozato O, Iizasa T, et al (2004). Antitumor effects produced by transduction of dendritic cells-derived heterodimeric cytokine genes in murine colon carcinoma cells. Anticancer Res, 24, 3763-7.

Chiyo M, Shimozato O, Yu L, et al (2005). Expression of IL-27 in murine carcinoma cells produces antitumor effects and induces protective immunity in inoculated host animals. Int 
$J$ Cancer, 115, 437-42.

Devergne O, Hummel M, Koeppen H, et al (1996). A novel interleukin-12 p40-related protein induced by latent EpsteinBarr virus infection in B lymphocytes. $J$ Virol, 70, 1143-53.

Di Carlo E, Sorrentino C, Zorzoli A, et al (2014). The antitumor potential of Interleukin-27 in prostate cancer. Oncotarget, 5, 10332-41.

Ge P, Xiao G (2016). Interleukin-27 rs153109 polymorphism and the risk of non-small-cell lung cancer in a Chinese population. Onco Targets Ther, 9, 895-8.

Ghavami A, Fathpour G, Amirghofran Z (2018). Association of IL-27 rs153109 and rs17855750 polymorphisms with risk and response to therapy in acute lymphoblastic leukemia. Pathol Oncol Res, 24, 653-62.

Guo J, Qin A, Li R, et al (2012). Association of interlenkin-27 gene polymorphism with genetic susceptibility to colorectal cancer. Chongqing Med, 41, 948-50.

Hisada M, Kamiya S, Fujita K, et al (2004). Potent antitumor activity of interleukin-27. Cancer Res, 64, 1152-6.

Huang ZQ, Wang JL, Pan GG, et al (2012). Association of single nucleotide polymorphisms in IL-12 and IL-27 genes with colorectal cancer risk. Clin Biochem, 45, 54-9.

Hunter CA, Kastelein R (2012). Interleukin-27: balancing protective and pathological immunity. Immunity, 37, 960-9.

Kastelein RA, Hunter CA, Cua DJ (2007). Discovery and biology of IL-23 and IL-27: related but functionally distinct regulators of inflammation. Annu Rev Immunol, 25, 221-42.

Lichtenstein P, Holm NV, Verkasalo PK, et al (2000). Environmental and heritable factors in the causation of cancer--analyses of cohorts of twins from Sweden, Denmark, and Finland. N Engl J Med, 343, 78-85.

Liu L, Wang S, Shan B, et al (2008). IL-27-mediated activation of natural killer cells and inflammation produced antitumour effects for human oesophageal carcinoma cells. Scand $J$ Immunol, 68, 22-9.

Liu Z, Liu JQ, Talebian F, et al (2013). IL-27 enhances the survival of tumor antigen-specific CD $8+\mathrm{T}$ cells and programs them into IL-10-producing, memory precursor-like effector cells. Eur J Immunol, 43, 468-79.

Lyu S, Ye L, Wang O, et al (2015). IL-27 rs153109 polymorphism increases the risk of colorectal cancer in Chinese Han population. Onco Targets Ther, 8, 1493-7.

Munretnam K, Alex L, Ramzi NH, et al (2014). Association of genetic and non-genetic risk factors with the development of prostate cancer in Malaysian men. Mol Biol Rep, 41, 2501-8.

Nagai H, Oniki S, Fujiwara S, et al (2010). Antitumor activities of interleukin-27 on melanoma. Endocr Metab Immune Disord Drug Targets, 10, 41-6.

Nie X, Yuan F, Chen P, et al (2017). Association between IL-27 gene polymorphisms and risk of papillary thyroid carcinoma. Biomark Med, 11, 141-9.

Owaki T, Asakawa M, Morishima N, et al (2005). A role for IL-27 in early regulation of Th1 differentiation. J Immunol, 175, 2191-200.

Pan G, Lu D, Liao L, et al (2012). Association of the genotype and serum level of IL-27 with nasopharyngeal carcinoma. Shandong Med J, 52, 18-20.

Peng Q, Qin X, He Y, et al (2013). Association of IL27 gene polymorphisms and HBV-related hepatocellular carcinoma risk in a Chinese population. Infect Genet Evol, 16, 1-4.

Pflanz S, Hibbert L, Mattson J, et al (2004). WSX-1 and glycoprotein 130 constitute a signal-transducing receptor for IL-27. J Immunol, 172, 2225-31.

Pflanz S, Timans JC, Cheung J, et al (2002). IL-27, a heterodimeric cytokine composed of EBI3 and $\mathrm{p} 28$ protein, induces proliferation of naive CD4+ T cells. Immunity, 16, 779-90.
Poudyal D, Herman A, Adelsberger JW, et al (2018). A novel microRNA, hsa-miR-6852 differentially regulated by Interleukin-27 induces necrosis in cervical cancer cells by downregulating the FoxM1 expression. Sci Rep, 8, 900.

$\mathrm{Pu}$ Y, Chen P, Zhou B, et al (2015). Association between polymorphisms in IL27 gene and renal cell carcinoma. Biomarkers, 20, 202-5.

Siegel RL, Miller KD, Jemal A (2015). Cancer statistics, 2015. CA Cancer J Clin, 65, 5-29.

Swaminathan S, Hu X, Zheng X, et al (2013). Interleukin-27 treated human macrophages induce the expression of novel microRNAs which may mediate anti-viral properties. Biochem Biophys Res Commun, 434, 228-34.

Tang YJ, Wang JL, Nong LG, et al (2014). Associations of IL-27 polymorphisms and serum IL-27p28 levels with osteosarcoma risk. Medicine (Baltimore), 93, e56.

Tao YP, Wang WL, Li SY, et al (2012). Associations between polymorphisms in IL-12A, IL-12B, IL-12Rbeta1, IL-27 gene and serum levels of IL-12p40, IL-27p28 with esophageal cancer. J Cancer Res Clin Oncol, 138, 1891-900.

Wang GQ, Zhao WH, Zhao XX, et al (2016). Association between IL-27 2905T/G genotypes and the risk and survival of cervical cancer: a case-control study. Biomarkers, 21, 272-5.

Wei YS, Lan Y, Luo B, et al (2009). Association of variants in the interleukin-27 and interleukin-12 gene with nasopharyngeal carcinoma. Mol Carcinog, 48, 751-7.

Yoshida H, Hunter CA (2015). The immunobiology of interleukin-27. Annu Rev Immunol, 33, 417-43.

Yoshimoto T, Chiba Y, Furusawa J, et al (2015). Potential clinical application of interleukin-27 as an antitumor agent. Cancer Sci, 106, 1103-10.

Yu XZ, Zhang Z, Zhou B, et al (2016). Genetic polymorphisms of interleukin-27 is associated with endometrial cancer susceptibility in Chinese Han women. Int J Clin Exp Pathol, 9, 2718-25.

Zhang S, Gao X, Wang Y, et al (2015). Interleukin $27-964 \mathrm{~A}>\mathrm{G}$ genetic polymorphism and serum IL-27p28 levels in Chinese patients with papillary thyroid cancer. Tumour Biol, 36, 8207-11.

Zhang S, Wang Y, Wang M, et al (2014a). IL-27 -964A>G polymorphism and the risk of breast cancer: a case-control study. Tumour Biol, 35, 12099-102.

Zhang Z, Zhou B, Wu Y, et al (2014b). Prognostic value of IL-27 polymorphisms and the susceptibility to epithelial ovarian cancer in a Chinese population. Immunogenetics, 66, 85-92.

Zhao B, Meng LQ, Huang HN, et al (2009). A novel functional polymorphism, $16974 \mathrm{~A} / \mathrm{C}$, in the interleukin-12-3' untranslated region is associated with risk of glioma. $D N A$ Cell Biol, 28, 335-41.

Zhou B, Zhang P, Tang T, et al (2015). Polymorphisms and plasma levels of IL-27: impact on genetic susceptibility and clinical outcome of bladder cancer. BMC Cancer, 15, 433.

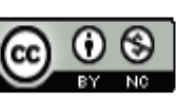

This work is licensed under a Creative Commons AttributionNon Commercial 4.0 International License. 